\title{
Gyrotron-backward-wave-oscillator experiments utilizing a high current, high voltage, microsecond electron accelerator
}

\author{
Thomas A. Spencer, Ronald M. Gilgenbach, and Jin J. Choia) \\ Intense Energy Beam Interaction Laboratory, Nuclear Engineering Department, University of Michigan, \\ Ann Arbor, Michigan 48109-2104
}

(Received 2 December 1991; accepted for publication 2 May 1992)

\begin{abstract}
We report the first gyrotron-backward-wave-oscillator experiments to produce high power (tube power of $\sim 1-8 \mathrm{MW})$; long-pulse $(0.3-1.2 \mu \mathrm{s})$ microwaves at high currents $(0.1-2 \mathrm{kA})$ and high voltages $(650-750 \mathrm{kV})$. Experiments were performed in the $\mathrm{TE}_{11}$ fundamental backward-wave mode, with efficiencies of $\sim 1 \%-2 \%$. Mode competition was observed which is believed to originate from the $\mathrm{TE}_{21}$ absolute instability.
\end{abstract}

Electron cyclotron resonance masers (ECMs) have the potential of producing high power microwaves for many applications such as fusion plasma heating, ${ }^{1}$ bulk material heating, ${ }^{2}$ and radar. Gyrotron-backward-wave oscillators (gyro-BWOs) make excellent high power devices due to the relative insensitivity to beam velocity spread and beam voltage fluctuations when compared to other electron cyclotron resonance devices like the gyro-traveling-wave tube, ${ }^{3,4}$ gyrotron, ${ }^{5}$ and cyclotron autoresonance maser (CARM) ${ }^{6}$ This insensitivity to beam velocity spread and voltage fluctuation allows the use of a low quality electron beam generated from explosive emission cathodes used in accelerators which produce gigawatts of $e$-beam power. Gyro-BWOs have the advantage of being fast-wave devices and employing a simple hollow tube. This leads to the possibility of enhanced power handling capabilities, since a periodic structure is not necessary as in conventional backward-wave-oscillator devices. Previous and recent gyro-BWO experiments have concentrated on low current $(<10 \mathrm{~A})$ and moderate voltage $(<100 \mathrm{kV}) e$ beams, and have obtained 1-10 kW of output power with efficiencies of up to $15 \% .^{7-10}$

In this article, we report the first gyro-BWO experiments employing a high voltage $(600-750 \mathrm{kV})$, high current (150-2000 A), long-pulse $(0.5-4 \mu \mathrm{s})$ electron beam.

The experimental configuration is shown in Fig. 1. The electron beam produced by the Michigan Electron Long Beam Accelerator (MELBA) ${ }^{11}$ is emitted from a cotton velvet cathode surface in a uniform magnetic field. The $e$ beam is extracted through a carbon anode, and then adiabatically compressed by the maser solenoidal coils as it propagates towards the interaction region. Experiments utilized either of two anodes. The first anode gave an annular $e$ beam of 150-300 A extracted through 24 holes of 5 $\mathrm{mm}$ in diameter drilled on a circle of $2.52 \mathrm{~cm}$ radius on a carbon plate. A second type of anode generated a solid $e$ beam of 1-2 kA extracted through a $5.1 \mathrm{~cm}$ diam hole. The maser solenoidal magnetic field and the smooth interaction tube are designed to excite the $\mathrm{TE}_{11}$ fundamental mode backward wave. The maser solenoidal coils, which generate a magnetic field of 3-7 kG, and the diode magnetic field

\footnotetext{
a) Present address: Science Applications International Corp., McLean, VA
} 22102. coils, with a magnetic field range of $0.4-0.9 \mathrm{kG}$, are independently pulsed to allow control over the $e$-beam velocity ratio, $\alpha=v_{1} / v_{\|}$. The interaction tube has a radius of 1.93 $\mathrm{cm}$, which sets a $T E_{11}$ mode cutoff frequency of $4.55 \mathrm{GHz}$, and a length of $50.5 \mathrm{~cm}$. After exiting the interaction region, the $e$ beam is deflected into the wall of the drift tube by a pair of permanent magnets. The backward wave generated in the interaction region is extracted at the diode end of the experiment by a modified $S$-band rectangular waveguide antenna, which is cut at a $30^{\circ}$ angle relative to the broad wall. The $S$-band antenna was calibrated with a low power microwave generator signal launched down the tube towards the antenna in a $\mathrm{TE}_{11}$ mode; this antenna collects a maximum of about $9 \%-11 \%$ of the tube power over the frequency range of $4.5-6.6 \mathrm{GHz}$. After extraction, the microwave signal travels through a $0.64 \mathrm{~cm}$ lucite window and through a waveguide detection system that consists of several different bands of rectangular waveguide and coaxial low pass filters to determine the frequency range of the interaction ( $G$ band, $f_{\mathrm{co}}=3.15 \mathrm{GHz} ; J$ band, $f_{\mathrm{co}}=4.30 \mathrm{GHz}$; and $H$ band, $f_{\mathrm{co}}=5.27 \mathrm{GHz}$ ). The signals are detected by coaxial diode detectors and displayed on fast oscilloscopes. A calorimeter is located at the downstream end of the experiment to measure the reflected backward-wave energy for comparison to that detected in the waveguide detection system located at the diode end. A copper guiding screen is used to direct the microwaves onto the calorimeter, which is placed $90^{\circ}$ off-axis so that the $e$ beam will not strike the calorimeter.

The uncoupled dispersion relation ${ }^{12}$ for an electron beam interacting with a waveguide can be obtained by simultaneously solving the waveguide vacuum modes

$$
\omega^{2}-\omega_{c 0}^{2}-k_{z}^{2} c^{2}=0
$$

and the beam cyclotron modes,

$$
\omega-k_{z} v_{z 0}-s \Omega_{r}=0,
$$

where $\omega_{\mathrm{c} 0}$ is the cavity cutoff frequency, $k_{z}$ is the axial wave number, $c$ is the speed of light, $v_{20}$ is the axial velocity of the beam, $s$ is the harmonic number ( $s=1$ is the fundamental mode), and $\Omega_{r}$ is the relativistic cyclotron resonance frequency, $\Omega_{r}=e B / \gamma m$ where $e$ is the electron charge, $B$ is the magnetic field, $\gamma$ is the relativistic factor, and $m$ is the mass of the electron. 


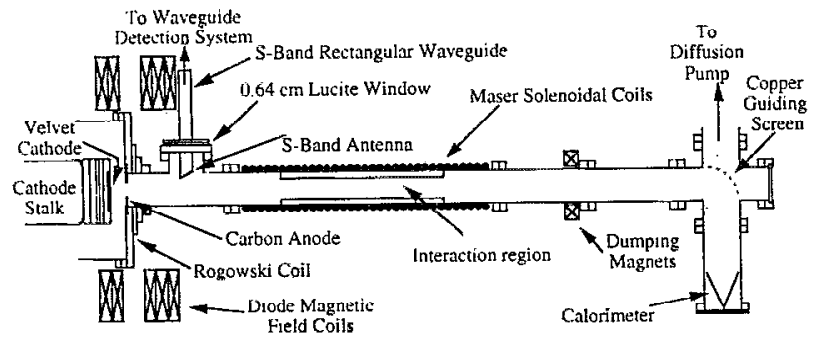

FIG. 1. Experimental configuration.

Figure 2 shows an uncoupled, e-beam-filled cavity dispersion relation that illustrates the $T E_{11}$ fundamental cyclotron mode $(s=1)$ and second harmonic $(s=2)$ backward-wave intersections, as well as the $\mathrm{TE}_{21}$ mode intersections for typical parameters used in the experiments: $V_{\text {beam }}=750 \mathrm{kV} ; I_{\text {beam }}=2 \mathrm{kA}, \alpha=0.65 ; B$ field $=6.3$ $\mathrm{kG}$.

Figure 3 depicts typical data obtained for the solid and annular $e$ beams. The top traces of Figs. 3(a) $-3(\mathrm{c})$ show accelerator voltage pulses. The bottom trace of Fig. 3(a), and the center traces of Figs. 3(b) and 3(c) show microwave data signals detected in the frequency range of $3.2 \leqslant f \leqslant 6.6 \mathrm{GHz}$. The bottom traces of Figs. 3(b) and 3(c) show microwave data signals detected in the frequency range of $5.3 \leqslant f<8 \mathrm{GHz}$. The center and bottom traces of Fig. 3(b) are on approximately the same scale. The center and bottom traces of Figs. 3(c) are on approximately the same scale [but not the same scale as those traces of Fig. 3 (b)]. Figure 3(a) depicts the measured $G$-band signal obtained for the solid beam $\left(I_{\text {beam }} \sim 1.5 \mathrm{kA}\right)$ at a magnetic field of $4.8 \mathrm{kG}$. Furthermore, the absence of microwave signal measured in an $H$-band waveguide $\left(f_{\mathrm{co}}=5.27 \mathrm{GHz}\right)$ coupled with an $8 \mathrm{GHz}$ low-pass filter demonstrated the measured signal existed within $3.2 \leqslant f \leqslant 5.3 \mathrm{GHz}$. The peak of the emission signal corresponds to $\sim 120 \mathrm{~kW}$ of extracted power, or, based on the calibration of the $S$-band

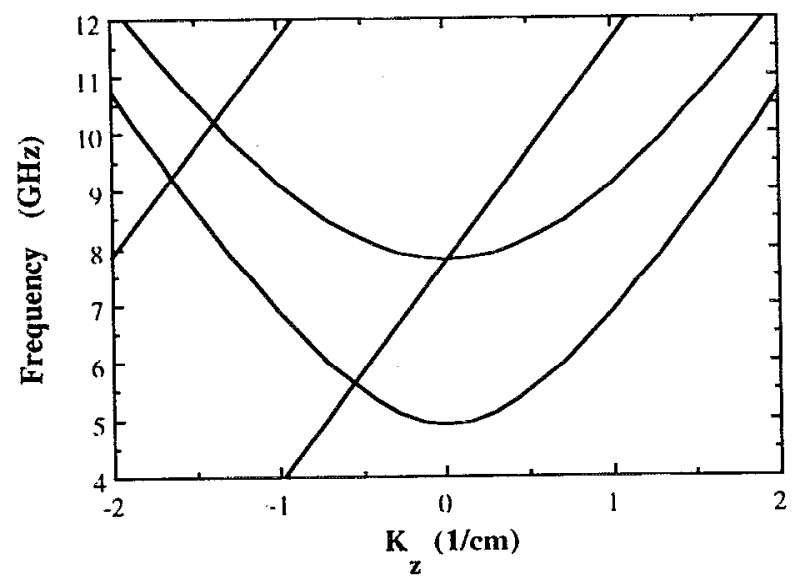

FIG. 2. The uncoupled, $e$-beam-filled cavity dispersion relations illustrating the fundamental $(s=1)$ and second $(s=2)$ harmonic intersections between the $\mathrm{TE}_{11}$ and $\mathrm{TE}_{21}$ cavity and $e$-beam modes. The parameters are: $V_{\text {beam }}=750 \mathrm{kV} ; I_{\text {beam }}=2 \mathrm{kA}, \alpha=0.5 ; B$ field $=6.3 \mathrm{kG}$. a)

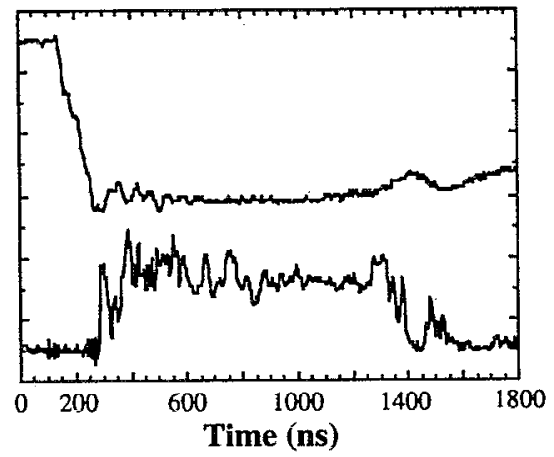

b)
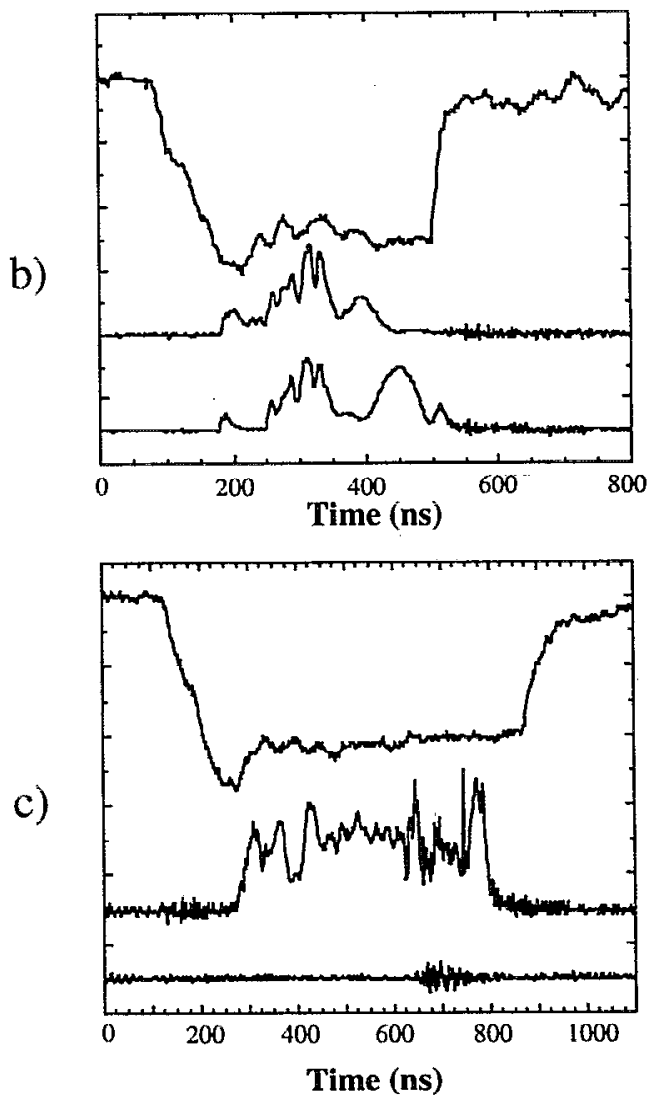

FIG. 3. Experimental data signals. (a) top trace: Voltage ( $310 \mathrm{kV} / \mathrm{div})$; bottom trace: microwave detector signal: $3.2 \leqslant f \leqslant 6.6 \mathrm{GHz}$, peak extracted power $\sim 120 \mathrm{~kW}$. (b) and (c) top trace: Voltage $(310 \mathrm{kV} /$ div); center trace: microwave detector signal: $3.2<f<6.6 \mathrm{GHz}$; bottom trace: microwave detector signal: $5.3<f<8 \mathrm{GHz}$. The center trace in (b) has a signal peak corresponding to $\sim 550 \mathrm{~kW}$ of extracted power, while the center trace in (c) has a signal peak of $\sim 80 \mathrm{~kW}$ of extracted power. (a) and (b) are data from the solid e-beam experiments, and (c) is data from the annular e-beam experiments.

antenna, $\sim 1.2 \mathrm{MW}$ of tube power. Figure 3(a) also shows that the emission signal remains within the frequency band throughout the flattop of the voltage pulse, $\sim 1.2 \mu \mathrm{s}$ in duration.

As the magnetic field is increased, the microwave signal is detected in both frequency ranges, as demonstrated in Fig. 3(b) $(B=6.6 \mathrm{kG})$, which shows the microwave data signal lies within $5.3<f<6.6 \mathrm{GHz}$. The peak of the microwave emission corresponds to $\sim 550 \mathrm{~kW}$ of extracted power (about 5.5 MW of tube power). Based on the data 
a)

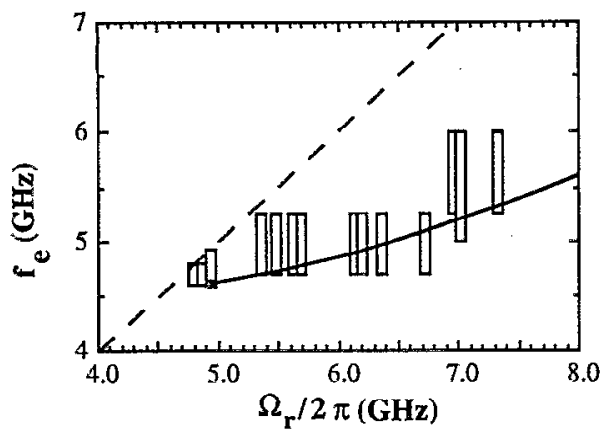

b)

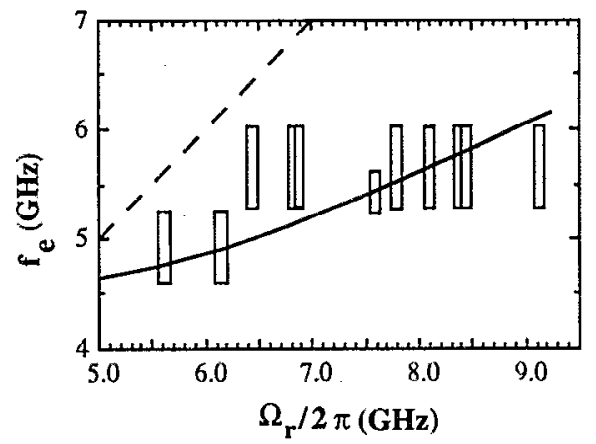

FIG. 4. The measured emission frequency as a function of the relativistic cyclotron frequency. The dashed line is the $f_{e}=\Omega_{r} / 2 \pi$ line. (a) is the solid beam data, where the solid line is the theoretical frequency for an uncoupled $e$-beam-filled dispersion relation for $\alpha=0.65$, and $I_{\text {beam }}=1.5$ $\mathrm{kA}$. (b) is the annular beam data, where the solid line is the theoretical frequency for $\alpha=0.65$ and $I_{\text {beam }}=300 \mathrm{~A}$.

given by Figs. 3(a) and 3(b), and the dispersion relations, the emission signals were determined to be the $\mathrm{TE}_{11}$ fundamental mode gyrotron-backward-wave interaction. The last peak of the microwave data signal in the bottom trace of Fig. 3(b) has a frequency range of $6.6 \leqslant f \leqslant 8 \mathrm{GHz}$, and could be the $\mathrm{TE}_{21}$ fundamental mode absolute instability (as shown in Fig. 2) competing with the fundamental $T E_{11}$ gyrotron-backward-wave interaction. Also observed (but not shown) in the solid beam experiments was shortpulsed, spiky emission believed to originate from the $\mathrm{TE}_{11}$ second harmonic gyrotron backward wave, with the extracted power approximately $10 \mathrm{~kW}$, less than a tenth of that seen for the fundamental $\mathrm{TE}_{11}$ mode. Figure 3(c) shows typical microwave signals for the annular beam ( $I_{\text {beam }} \sim 200 \mathrm{~A}, B=5.0 \mathrm{kG}$ ) which demonstrate long-pulse microwaves of $\sim 630 \mathrm{~ns}$ in the frequency range of 3.2 $\leqslant f \leqslant 5.3 \mathrm{GHz}$. The emission signal peak corresponds to $\sim 80 \mathrm{~kW}$ of extracted microwave power $(\sim 0.8 \mathrm{MW}$ of tube power), about one-tenth of that observed with the solid beam.

Proof that the interaction is the $\mathrm{TE}_{11}$ fundamental backward-wave interaction is demonstrated in two ways. The first is that the emission frequency is shown to be less than that of the cyclotron resonance frequency $\left(\Omega_{n} / 2 \pi\right)$ for both the solid and annular $e$ beams, as shown in Fig. 4. The solid line in Fig. 4(a) gives the theoretical frequency for an uncoupled $e$-beam-filled cavity dispersion relation with $\alpha=0.65$, and $I_{\text {beam }}=1.5 \mathrm{kA}$. The solid line in Fig. 4 (b) gives the theoretical frequency with $\alpha=0.65$ and $I_{\text {beam }}$ $=300 \mathrm{~A}$. The dashed line in Figs. $4(\mathrm{a})$ and $4(\mathrm{~b})$ is the

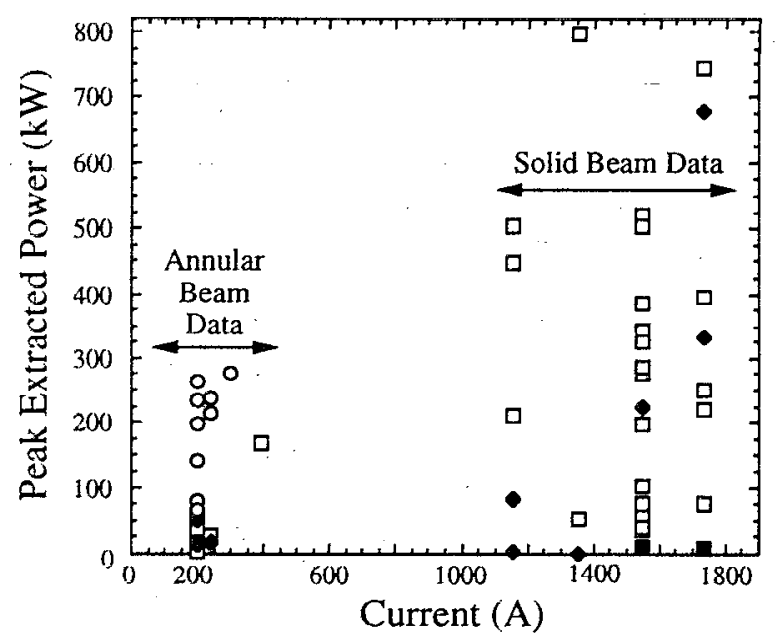

FIG. 5. Microwave peak extracted power as a function of the beam current for the annular and solid electron beams. $\square$ are the measured data points where: $3.2 \leqslant f<6.6 \mathrm{GHz}$; are the measured data points where: $5.3<f<8 \mathrm{GHz}$; and $O$ are the measured data points obtained during the initial voltage overshoot where: $3.2<f<6.6 \mathrm{GHz}$. ( $\square$ represent overlapping and $\square$.)

emission frequency equal to the cyclotron resonance frequency $\left(f_{e}=\Omega_{r} / 2 \pi\right)$. Note that all the measured frequencies lie below this line and thus the interaction is the backward wave. For the annular beam, the value of $\alpha$ has been determined from both Cerenkov emission and radiation darkening of a glass plate, ${ }^{13}$ and from the EGUN code, ${ }^{14}$ and is determined to lie between 0.5 and 0.7. For the solid $e$ beam, the spread in alpha is obtained from the EGUN code and is large, from 0.05 to 0.7 . The second way to demonstrate the existence of the backward wave is that the frequency of interaction increases as the magnetic field increases, as shown in Figs. 3(a), 3(b), and 4.

Figure 5 shows a summary of the annular and solid beam data. The solid beam provided the highest extracted powers $(300-800 \mathrm{~kW})$, typically five to ten times higher than that of the annular beam (10-80 kW) with similar $e$ beam and magnetic field parameters. The solid $e$ beam is expected to couple more strongly to the $\mathrm{TE}_{11}$ mode which has a peak of the electric field on-axis. For the annular beam, higher extracted powers (depicted by open circular data points in Fig. 5) were obtained for higher electron beam voltages $(850-950 \mathrm{kV}$ ) corresponding to the initial overshoot of the voltage pulse. This increased energy with the annular beam is due to the electrons having more perpendicular energy at higher beam voltages to amplify the electromagnetic waves. Based on the value of $I_{\text {beam }}$ for the solid and annular $e$ beams, the efficiencies are determined to be approximately the same (about $1 \%-2 \%$ electronic efficiency), demonstrating the gyro-BWO is insensitive to the $e$-beam energy spread. The relatively low efficiency may be due to the low $\alpha$ and space-charge limiting effects, or reabsorption of microwave power in the tube region. Saturation effects at high currents have been previously observed in Russian gyrotron experiments. ${ }^{15}$

Tube power estimates obtained from the $S$ - band antenna were consistent with those obtained from the calo- 
rimeter. Power estimates from the calorimeter (although the calorimeter data showed large fluctuation) were 7.9 MW (with a standard deviation of $3.4 \mathrm{MW}$ ) for the solid beam. This estimate was obtained by averaging calorimetry signals for all shots, at all magnetic fields which produced microwave signals on the diode detectors. The calorimeter data also showed the same general trend (increasing or decreasing) as the diode detector signals. The tube power not extracted by the $S$-band antenna was mostly absorbed by the calorimeter.

In summary, the gyro-BWO has been shown to produce high power (tube power of $\sim 1-8 \mathrm{MW}$ ), long-pulse $(0.3-1.2 \mu \mathrm{s})$ microwaves at high currents $(0.1-2 \mathrm{kA})$ and high voltages $(650-750 \mathrm{kV})$ in the $\mathrm{TE}_{11}$ fundamental backward-wave mode, with efficiencies of about 1-2\%. Also observed was possible mode competition from the $\mathrm{TE}_{21}$ absolute instability, and short-pulse emission believed to originate from the $\mathrm{TE}_{11}$ second harmonic backwardwave interaction.

This research was supported by the Air Force Office of Scientific Research and the Air Force Phillips Laboratory. Thomas Spencer was supported by an Air Force Laboratory Graduate Fellowship. We thank P. R. Menge and C. $\mathrm{H}$. Ching for experimental assistance.
${ }^{1}$ R. M. Gilgenbach, et al., Phys. Rev. Lett. 44, 647 (1980).

${ }^{2} \mathrm{~J}$. H. Booske (private comunication).

${ }^{3}$ Y. Y. Lau, K. R. Chu, L. R. Barnett, and V. I. Granatstein, Int. J. Infr. Millimeter Waves 2, 373 (1981).

${ }^{4}$ S. H. Gold, D. A. Kirkpatrick, A. W. Fliffet, R. B. McCowan, A. K. Kinkead, D. L. Hardesty, and M. Sucy, J. Appl. Phys. 69, 6696 (1991).

${ }^{5} \mathrm{~J}$. L. Hirshfield, Infrared and Millimeter Waves, Vol. 1 (Academic, New York, 1979).

${ }^{6}$ G. Bekefi, A. DiRienzo, C. Leibovitch, and B. G. Danly, Appl. Phys. Lett. 54, 1302 (1989).

${ }^{7}$ S. Y. Park, V. L. Granatstein, and R. K. Parker, Int. J. Electron. 57, 1109 (1984).

${ }^{8}$ S. Y. Park, R. H. Keyser, C. M. Armstrong, R. K. Parker, and V. L. Granatstein, IEEE Trans. Plasma Sci. PS-18, 321 (1990).

${ }^{9}$ P. Muggli, M. Q. Tran, and T. M. Tran, Phys. Fluids B 3, 1315 (1991).

${ }^{10}$ W. C. Guss, K. E. Kriescher, R. J. Temkin, M. Caplan, and D. Pirkle, Proceedings of the 14th International Conference on Infrared and Millimeter Waves, 1989 (SPIE, Bellingham, WA, 1989), Vol. 1240, p. 369.

${ }^{11}$ R. M. Gilgenbach, L. D. Horton, R. F, Lucey, Jr., S. Bidwell, M. Cuneo, J. Miller, and L. Smutek, in Digest of Technical Papers of the Fifth IEEE Pulsed Power Conference, Arlington, VA, 1985 (IEEE, New York, 1985), Catalog No. 85C2121-2.

${ }^{12}$ A. W. Fliflet, Int. J. Electron. 61, 1049 (1986).

${ }^{13}$ J. J. Choi, R. M. Gilgenbach, T. A. Spencer, P. Menge, and C. H. Ching, Rev. Sci. Instrum. 63, 1671 (1992).

${ }^{14}$ W. Hermannsfeldt, SLAC Report No. 226, 1979.

${ }^{15}$ N. S. Ginzberg, V. I. Krementsov, M. I. Petelin, P. S. Strelkov, and A. K. Shkvarunets, Sov. Phys. Tech. Phys. 24, 218 (1979). 